\title{
BEAM LOADING COMPENSATION REQUIREMENT FOR MULTIBATCH COALESCING IN FERMILAB MAIN INJECTOR
}

\author{
J. Dey, K. Koba, I. Kourbanis, J. Reid, FNAL, Batavia, IL 60510, USA
}

\section{Abstract}

Fermilab collider Run IIa requires 36 proton bunches with intensities 270E9ppb and 36 antiproton bunches with intensities 40-70E9ppb[1]. Currently the proton bunches are produced by coalescing $5-753 \mathrm{MHz}$ bunches into one $53 \mathrm{MHz}$ bunch and repeating this process a total of 36 times. It is necessary to coalesce each group of 5-7 bunches (called a 'batch') on independent cycles mainly because of beam loading. The beam loading requirements that would allow us to coalesce 4 proton batches at a time are presented.

\section{INTRODUCTION}

For the Run IIa operation, Main injector has to extract 1-4 proton batches every cycle. Each batch consists of 5 bunches which are concentrated into one bunch with a coalescing process. The intensity of the final proton bunch is $270 \mathrm{E} 9 \mathrm{ppb}$. During the coalescing, the $53 \mathrm{MHz} \mathrm{RF}$ voltage is reduced to $0-30 \mathrm{kV}$, therefore the beam loading effects are large.

We have already implemented the RF feedback system with a gain of $20 \mathrm{~dB}[2]$. In this paper, we will present beam loading measurement results, simulation results and discuss how much the Q-value must be reduced to avoid the beam loading effects for Run IIa.

\section{RF CAVITY IN MAIN INJECTOR}

Four kinds of cavities are used for Main Injector operation. The Main Injector radius is $528.3 \mathrm{~m}$, and so the revolution frequency is $90 \mathrm{kHz}$.

We are mainly using the $53 \mathrm{MHz}$ RF cavities for acceleration, which have an harmonic number of 588 . There are 18 stations and they are separated into two groups of 9 stations. By individually changing the voltage of each group of stations, the total amplitude of the RF voltage may be adjusted.

To extract the proton and antiproton beam at $2.5 \mathrm{MHz}$ to the Tevatron, five $2.5 \mathrm{MHz} \mathrm{RF}$ cavities are used and operated for coalescing.

We also have a $106 \mathrm{MHz}$ and a $5 \mathrm{MHz}$ cavity, higher harmonic cavities for the $53 \mathrm{MHz}$ and $2.5 \mathrm{MHz}$ systems, respectively.

Thus, the beam goes though many cavities and may be affected by their impedance. The $53 \mathrm{MHz}$ cavity has a large shunt impedance, and the fundamental frequency of the beam is $53 \mathrm{MHz}$, therefore the major beam loading effect on the beam should be due to these $53 \mathrm{MHz}$ cavities. In this paper, we will consider about beam loading effects caused by the $53 \mathrm{MHz}$ RF cavities only.

\section{MEASUREMENT OF THE BEAM LOADING EFFECTS}

During the coalescing, we first reduce the $53 \mathrm{MHz}$ RF voltage from $1.1 \mathrm{MV}$ to $30 \mathrm{kV}$. After a quarter of a synchrotron period at $30 \mathrm{kV}$, the $53 \mathrm{MHz} \mathrm{RF}$ voltage is reduced close to zero by paraphasing each group of stations by 90 degrees, with each group moving oppositely in phase. Then the bunches rotate $60 \mathrm{kV}$ bucket of the $2.5 \mathrm{MHz} \mathrm{RF}$.

We estimated the beam loading effects for the case of 4 batches, with each batch consisting of 5 bunches. The total intensity was taken to be $0.18 \mathrm{E} 12$.

If we assume that the bunch length is short, the induced voltage is can be written in equation (1)[3].

$$
V_{b l}(n)=\frac{q \omega_{r f} R}{Q} e^{-n \pi / Q}
$$

where $q$ is the charge per bunch, $R$ is the shunt impedance of the RF cavity, $\omega_{r f}$ is the resonant angular frequency, $Q$ is the $\mathrm{Q}$-value of the cavity and $n$ is the bunch spacing given in number of buckets.

Since there are $18 \mathrm{RF}$ stations in the Main Injector, the total induced voltage is $V_{b l}($ total $)=18 \times V_{b l}$. The $R / Q$ is 100 for each station, and the Q-value is 500 when RF feedback is on. The calculation results of the total induced voltage and phase shift which is compared with the $30 \mathrm{kV}$ of applied RF voltage in Fig. 1.

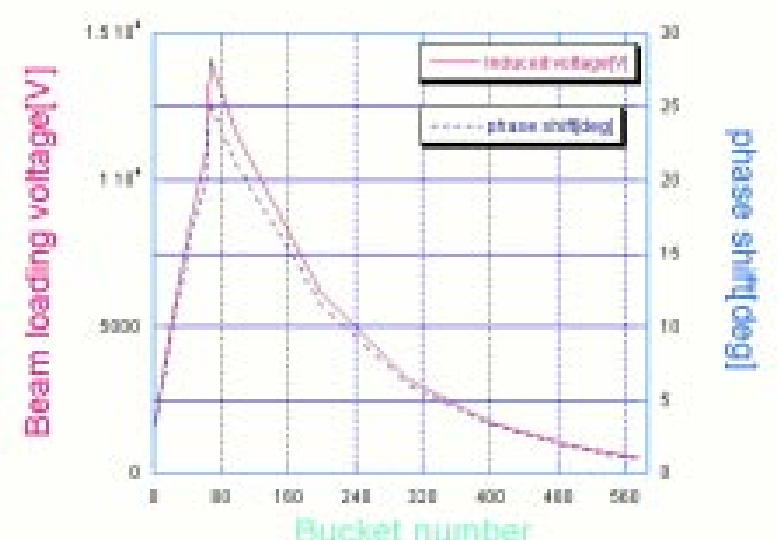

Figure 1: The calculation results of the total induce voltage and phase shift which is compared with $30 \mathrm{kV}$ of applied RF voltage 
We have measured these effects by using a phase detector and gap voltage monitor. Based on the calculation, we expected to measure a voltage of about $15 \mathrm{kV}$ and a phase shift of $25 \mathrm{deg}$. When the bunches are in a $30 \mathrm{kVbucket}$ of the $53 \mathrm{MHz}$ system, it was not easy to determine the induce voltage from the gap monitor, because the induce voltage should be almost same as the $\mathrm{RF}$ voltage. On the other hand, since the voltage of the $53 \mathrm{MHz} \mathrm{RF}$ is zero when bunches are captured by $2.5 \mathrm{MHz}$ RF voltage, we can't compare this phase with the phase of $53 \mathrm{MHz}$ RF voltage.

Figure 2 and 3 show the phase detector signal when the $53 \mathrm{MHz} \mathrm{RF}$ voltage was $30 \mathrm{kV}$, and the gap voltage monitor signal when the $53 \mathrm{MHz}$ RF voltage was $0 \mathrm{~V}$.

Figure 2 shows a maximum phase shift of 23.5 degrees which agrees with the calculation results. We also estimated the Q-value using the decay rate, and found it was 480 . From Fig. 3, the maximum gap voltage is $8.2 \mathrm{kV}$.

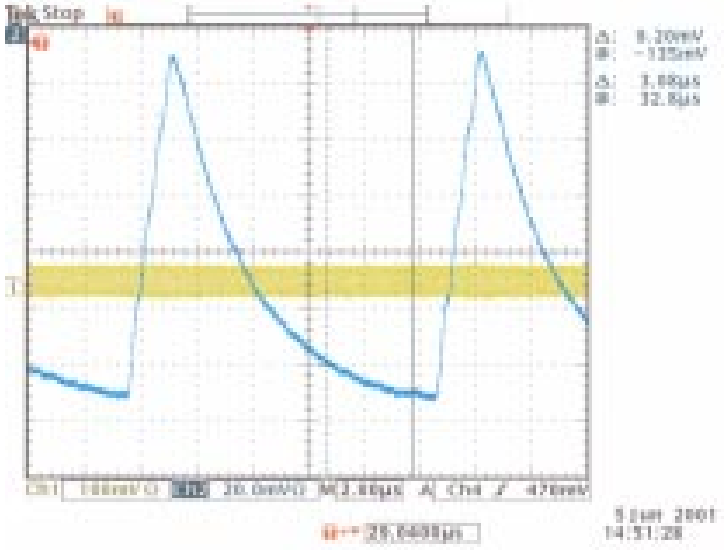

Figure 2: RF gap voltage phase shift detected by phase detector (189 degrees/volt). Scale of the voltage is $20 \mathrm{mV} / \mathrm{div}$ and time is $2.0 \mu \mathrm{sec} / \mathrm{div}$

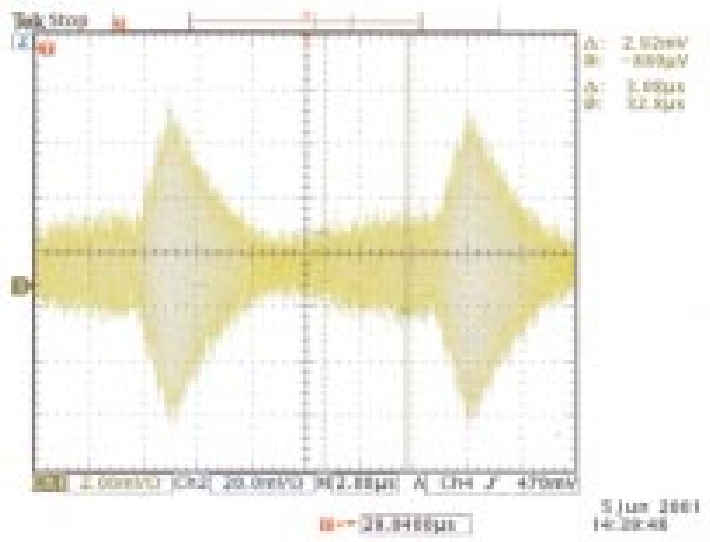

Figure 3: RF gap voltage $(1.2 \mathrm{kV} / 1 \mathrm{mV})$. Scale of the voltage is $2 \mathrm{mV} / \mathrm{div}$ and the time is $2.0 \mu \mathrm{sec} / \mathrm{div}$

\section{BEAM MEASUREMENT AND SIMULATION}

We also measured the bunch shape during the coalescing to know how the beam loading affects to the beam. These are Mt. range plots for the cases of low intensity $\left(0.12 * 10^{12} \mathrm{ppp}\right)$ and high intensity $\left(0.42 * 10^{12} \mathrm{ppp}\right)$ are shown in Fig. 4 and 5, respectively.

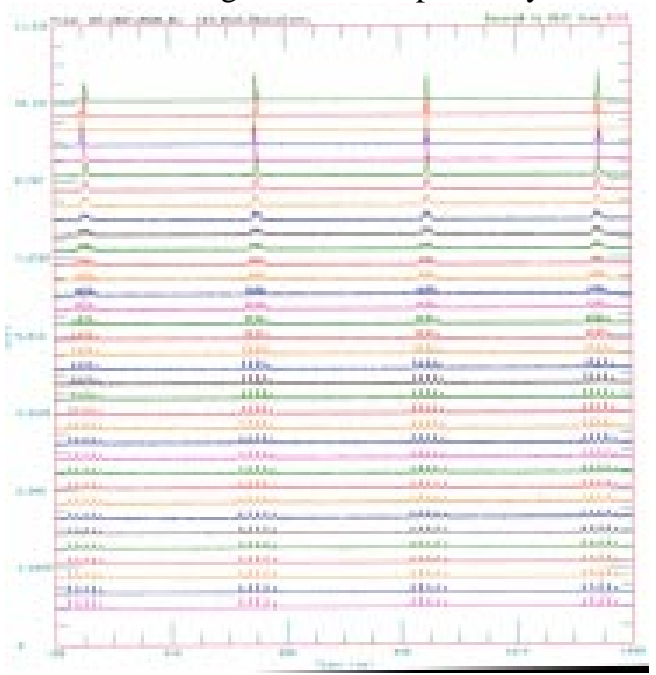

Figure 4: Measurement results in the case of the intensity is $0.12 * 10^{12}$ particles per pulse.

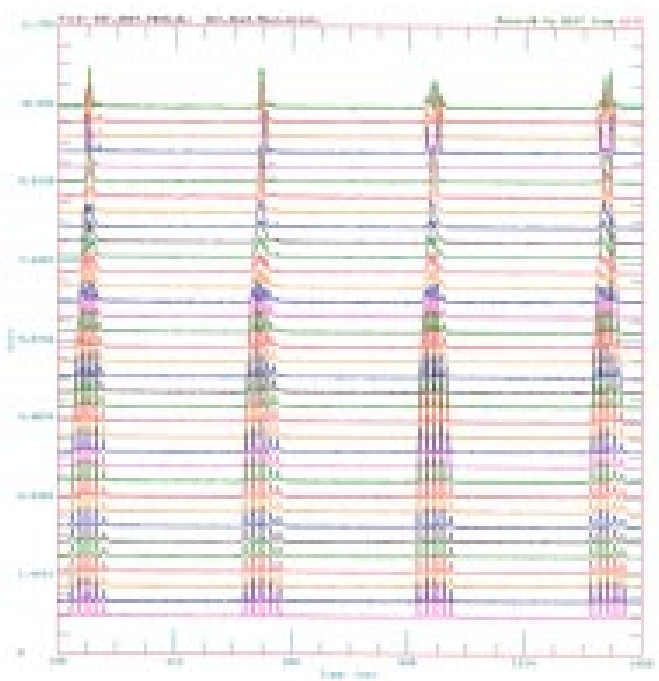

Figure 5: Measurement results in the case of the intensity is $0.42 * 10^{12}$ particles per pulse.

Multi particle ESME[4] simulations were carried out with beam loading effects and having the same intensities as the measurements. Figure 6 and 7 show mountain range plots obtained by simulation. In the low intensity case, there is no emittance growth after coalescing. On the other hand, in high intensity case, we can see emittance growth clearly in the both the measurement and in the simulation results. The simulation results are in good agreement with the measurement results. 
Beam Current Profiles

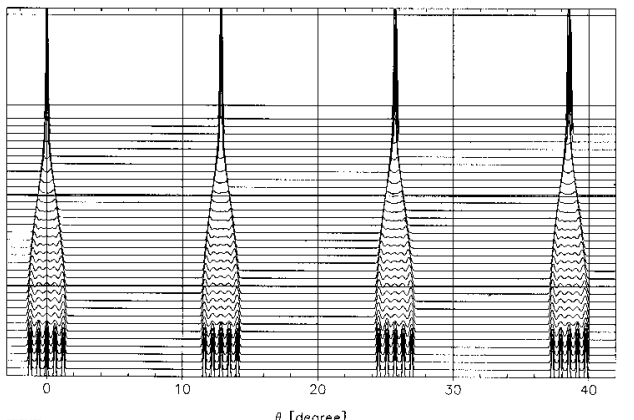

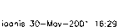

Figure 6: Simulation results with the intensity of $0.12 * 10^{12}$ particles per pulse and $\mathrm{Q}=500$.

$$
\begin{aligned}
& \text { CAPTURE WITH 1MV } \\
& \text { every } 200 \text { turns, from turn } 200
\end{aligned}
$$

Beom Current Profiles

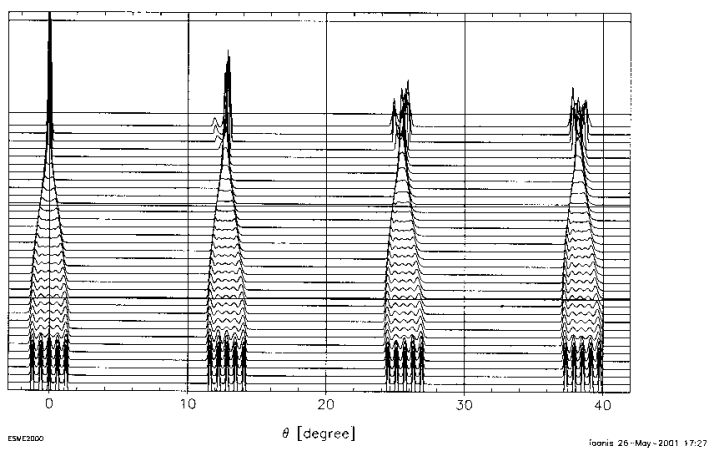

Figure 7: Simulation results with the intensity of $0.42 * 10^{12}$ particles per pulse and $\mathrm{Q}=500$.

\section{SIMULATION FOR THE RUN IIA GOAL}

In near future, proton bunch with $270 \mathrm{E} 9$ particles will be required for Run II operation. There is also requirement to coalesce 4 proton batches on the same machine cycle at this high intensity. Thus, the intensity will be 4 times higher than for the case which we presented in section 3. Therefore the beam loading compensation is a most important issue for Main Injector requirement.

In order to optimize the feed back gain, the multi particle simulation was carried out with required beam parameters, and the results are shown in Fig. 8 and 9. According to the results, the gain of the feedback must be 4 times larger for Run II operation. We have already stated development of the feed ford system to compensate the beam loading effects, and the Q value is supported to be around 25 .

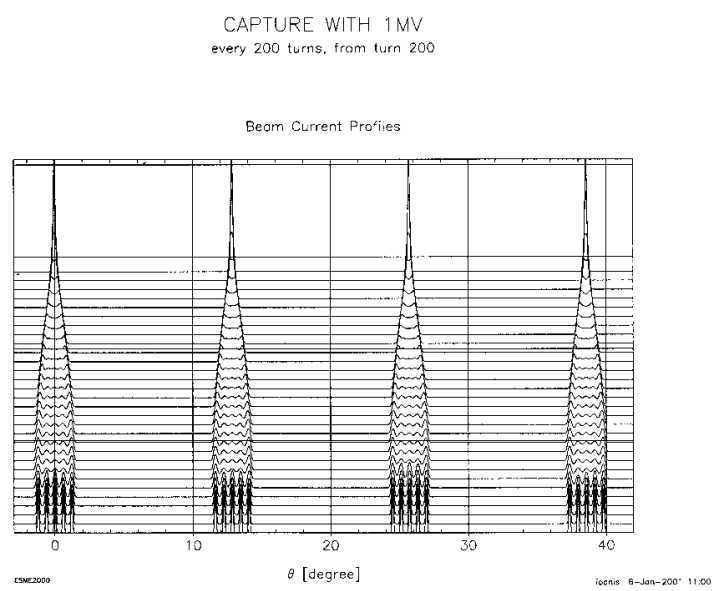

Figure 8: Simulation results with the intensity of $1.2 * 10^{12}$ particles per pulse and $\mathrm{Q}=25$.

4 BATCHES 5 BUNCHES $0.000 E+O D O$
TUEC

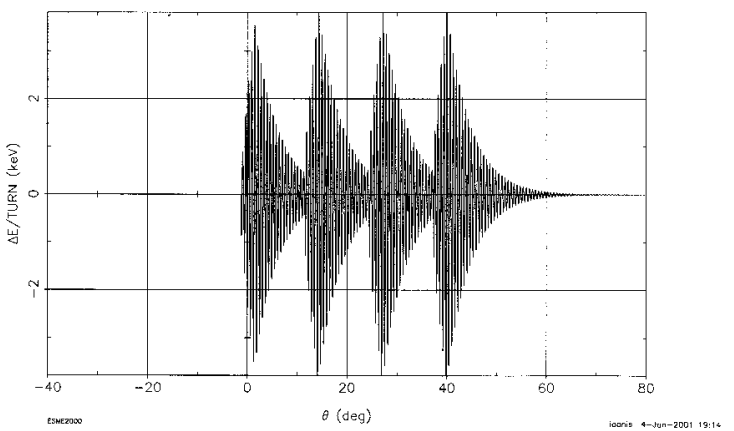

Figure 9: The beam loading voltage obtained by the simulation with the intensity of $1.2 * 10^{12}$ particles per pulse.

\section{CONCLUTION}

The beam loading effects for the case of 4 proton batches was measured, calculated, and simulated with the intensity of around 180Eppb. For the collider Run IIa operation, which has a required intensity $270 \mathrm{E} 9 \mathrm{ppb}$ protons, the $\mathrm{Q}$ value is supposed to be around 25 .

\section{REFERENCES}

[1] K. Koba et al, "Status of the Fermilab Main Injector", these proceedings.

[2] J.Dey and J.Steimel, "Improving the linearity of ferrite loaded cavities using feedback", these proceedings

[3] J. Marriner, "Main Injector beam loading in the 2.5MHz system", not published.

[4] J. MacLachlan, "Users Guide to ESME”, 2000. 